\title{
Lagrangian mechanics without ordinary differential equations
}

\author{
George W. Patrick
}

September 2005

\begin{abstract}
A variational proof is provided of the existence and uniqueness of evolutions of regular Lagrangian systems.
\end{abstract}

\section{Introduction}

Let $\mathcal{Q}$ be a smooth, finite dimensional manifold and $L: T \mathcal{Q} \rightarrow \mathbb{R}$ be a smooth Lagrangian. Evolutions of the Lagrangian system defined by $L$ are by definition the $C^{1}$ curves $q:[0, h] \rightarrow \mathbb{R}$ which are critical points of the action

$$
S_{h}=\int_{0}^{h} L \circ q^{\prime}(t) d t
$$

subject to the constraint that $q(0)$ and $q(h)$ are constant. A typical route to existence and uniqueness (given that $L$ is regular) of the Lagrangian evolutions, is to to show that derivatives $q^{\prime}(t)$ of evolution curves $q(t)$ are integral curves of the Lagrangian vector field $X_{E}$, constructed either using the Euler-Lagrange equations in charts, or using the Lagrange two-form $\omega_{L}$, the energy $E$, and the equation

$$
\boldsymbol{i}_{X_{E}} \omega_{L}=\boldsymbol{d} E \text {. }
$$

In any case, standard ODE theory provides existence and uniqueness of the initial value problem $q(0)=q_{0}, q^{\prime}(0)=v_{0}$. For a self-contained exposition, see [1.

Given two nearby $q_{1}, q_{2} \in \mathcal{Q}$, does there exist a unique evolution curve $q(t)$ such that $q(0)=q_{1}$ and $q(h)=q_{2}$ ? This is the local boundary value problem of Lagrangian mechanics. The problem crops up in a variety of situations. For example:

1. If $\mathcal{Q}$ is a Riemannian manifold and $L(v)=\frac{1}{2} g(v, v)$, then the Lagrangian evolution curves are constant speed reparameterizations of the geodesics, and the local boundary value problem becomes that of locating the unique local geodesic connecting two sufficiently nearby points.

* Partially supported by the Natural Sciences and Engineering Research Council, Canada. 
2. A solution to the local boundary value problem is required to construct type 1 generating functions $S_{t}\left(q_{2}, q_{1}\right)$ for the Hamiltonian flow, which are defined by

$$
S_{t}\left(q_{2}, q_{1}\right)=\int_{0}^{t} L \circ q(t) d t
$$

where $q(t)$ is the evolution curve with $q(0)=q_{1}, q(t)=q_{2}$.

After constructing the Lagrangian flow $F_{t}^{X_{E}}$, the solution to the local boundary value problem is obtained by solving the equations

$$
\tau_{\mathcal{Q}} F_{t}^{X} X_{E}\left(v_{q_{1}}\right)=q_{2}
$$

for $v_{q_{1}} \in T_{q_{1}} \mathcal{Q}$ as a function of $q_{1}, q_{2}, t$, where $\tau_{\mathcal{Q}}: T \mathcal{Q} \rightarrow \mathcal{Q}$ is the canonical projection. This may appear to be a straightforward application of the implicit function theorem near $t=0, q_{1}=q_{2}$, but that is not quite so, because the equation fails to be appropriately regular there. With some care, however, the local boundary value problem can be solved by this route $[\underline{6}$.

But, first solving the initial value problem seems like a rather circuitous route to the solution of the local boundary value problem, especially considering that the boundary values $q_{1}$ and $q_{2}$ actually occur as the constraints in the original variational formulation for the evolution. Regular constrained optimization problems have critical points which persist as smooth functions of the constraint values. Why not solve the boundary value problem directly, avoiding an excursion into the initial value problem via ODE theory?

The obstruction to simply getting on with the job is a fundamental one: the problem of finding the critical points of $S_{h}$ subject to the constraint $q(0)=q_{0}$, $q(t)=q_{1}$, is nonregular at $h=0$, which precisely where one wants to perturb from. Indeed, the objective function $S_{h}$ is actually zero when $h=0$. More seriously, the constraint

$$
q(t) \mapsto(q(0), q(h))
$$

maps into the diagonal of $\mathcal{Q} \times \mathcal{Q}$ at $h=0$, and hence cannot be, even formally, a submersion. One cannot perturb from such a degenerate landscape.

In this article, I provide a direct variational proof of the local boundary value problem, using a regularization procedure, which is adapted from the one used in [3] for a similar problem in the context of discrete Lagrangian systems. The regularization procedure culminates in the replacement of the variational problem with an equivalent, regular one, after which readily available techniques used to prove the infinite dimensional Morse Lemma, and the (infinite dimensional) implicit function theorem, give the result.

\section{Regularization}

Assume that $L: \mathcal{Q} \rightarrow \mathbb{R}$ is $C^{r}$ with $r \geq 2$. Since the aim is to provide a local result, also assume that $\mathcal{Q}$ is an open subset of $\mathbb{R}^{n}$, and that $\bar{q} \in \mathcal{Q}$ is given. 
One seeks a perturbative approach from $t=0, q_{1}=q_{2}=\bar{q}$, so that $q_{1}$ and $q_{2}$ will be near $\bar{q}$. The regularization procedure is, step-by-step, as follows:

1. Transform the variational problem from one for curves in $\mathcal{Q}$ to one for curves in $T \mathcal{Q}$, with an additional first order constraint. The transformation is simply to seek critical points $(q(t), v(t)) \in T \mathcal{Q}$ of the objective

$$
S_{h}=\int_{0}^{h} L(q(t), v(t)) d t
$$

subject to the constraints

$$
v(t)=\frac{d q}{d t}, \quad q(0)=q_{1}, \quad q(h)=q_{2} .
$$

There are additional freedoms inherent in the use of curves in $T \mathcal{Q}$ rather than curves in $\mathcal{Q}$, and these will be important for the regularization.

2. Reparameterize, so that the solutions curves, which are defined on $[0, h]$, do not disappear as $h \rightarrow 0^{+}$. The curves $(q(t), v(t)), t \in[0, h]$ are replaced by the curves $(Q(t), V(t)), t \in[0,1]$ through

$$
Q(u)=q(h u), \quad V(u)=v(h u), \quad u \in[0,1] .
$$

For $h>0$, the new curve $(Q(u), V(u))$ satisfies an equivalent variational problem, which can be worked out as follows. First, substitute $u=t / h$ and divide by $h$ to obtain

$$
\frac{1}{h} \int_{0}^{h} L(q(t), v(t)) d t=\int_{0}^{1} L(Q(u), V(u)) d u .
$$

Since $h$ is constant for the variational principle, one can use the right-handside as an objective for $(Q(u), V(u))$. The first order constraint transforms as follows:

$$
\frac{d}{d t} q(t)-v(t)=\left(\frac{1}{h} \frac{d}{d u}(Q(u))-V(u)\right)_{u=\frac{t}{h}} .
$$

Multiplying this by $h$, the reparameterized variational principle is for curves $(Q(u), V(u))$ with values in $T \mathcal{Q}$ which are critical points of the objective

$$
S=\int_{0}^{1} L(Q(u), V(u)) d u,
$$

subject to the constraints

$$
\frac{d}{d u}(Q(u))-h V(u)=0, \quad Q(0)=q_{1}, \quad Q(1)=q_{2} .
$$

Notice that both the objective and the constraints are smooth through $h=0$ and that, at $h=0$, the first order constraint is equivalent to the constraint that the curve $(Q(u), V(u))$ lies in a fiber of $T \mathcal{Q}$ i.e. the curve is vertical. 
3. Restrict the remaining (boundary) constraints to the submanifold defined by the regularized first order constraint, and regularize the result. The first order constraint may be solved smoothly through $h=0$ by integration:

$$
Q(u)=Q(0)+h \int_{0}^{u} V(s) d s .
$$

Thus the set of curves $(Q(u), V(u))$ may be replaced by the set of curves $\{V(u)\}$. Also, $q_{2}$ may be replaced by $q_{1}+h z, z \in \mathbb{R}^{n}$, and then, since under (11),

$$
Q(1)=Q(0)+h \int_{0}^{1} V(s) d s,
$$

the objective and the boundary constraint become, respectively,

$$
S_{h}=\int_{0}^{1} L\left(q_{1}+h \int_{0}^{u} V(s) d s, V(u)\right) d u, \quad \int_{0}^{1} V(u) d u=z,
$$

while $h$ and $q_{1}$ appear as parameters.

This completes the procedure, since the variational problem (2) is formally regular through $h=0$. Indeed, at $h=0$, (2) becomes finding the curves $V(u)$ which are critical points of the constrained problem

$$
S_{0}=\int_{0}^{1} L\left(q_{1}, V(u)\right) d u, \quad \int_{0}^{1} V(u) d u=z .
$$

Using a Lagrange multiplier $\lambda$, the solutions are given by setting

$$
\int_{0}^{1} \frac{\partial L}{\partial v}\left(q_{1}, V(u)\right) \delta V(u) d u=\int_{0}^{1} \lambda \cdot \delta V(u) d u
$$

for all $\delta V(u)$ i.e.

$$
\frac{\partial L}{\partial v}\left(q_{1}, V(u)\right)=\lambda
$$

If $L$ is a regular Lagrangian then this implies $V(u)$ is constant, and the constraint then implies $V(u)=z$. Thus the solution to (2) at constraint value $z$ is the constant curve $z$, and there is exactly one critical point for each constraint value.

\section{Implicit function theorem solution to the reg- ularized variational problem}

Consider $S_{h}$ from (2) on the Banach space $C^{k}\left([0,1], \mathbb{R}^{n}\right)$ of curves $V(u)$, where $0 \leq k \leq r$. Since $L$ is $C^{r}$, the Omega Lemma (2], page 102) implies that the integrand of $S_{h}$ is $C^{r}$ as a map into $C^{0}([0,1], \mathbb{R})$. Since integration on $C^{0}([0,1], \mathbb{R})$ is bounded linear (and therefore $C^{\infty}$ ), it follows that $S_{h}$ is $C^{r}$, irrespective of the value of $k$. 
I specialize the method for proving the infinite dimensional Morse lemma 4, [7, 8] to the constrained variational problem (11). One first calculates the gradient of $S$ from the derivative $\boldsymbol{d} S_{h}$ using the $C^{2}\left([0,1], \mathbb{R}^{n}\right)$ weak inner product

$$
\left\langle\langle V, W\rangle=\int_{0}^{1} V \cdot W .\right.
$$

That computation is as follows (for short, below $Q(u)$ means the right side of (1)):

$$
\begin{aligned}
\boldsymbol{d}_{h}([ & {[V(u)]) \delta V(u) } \\
& =\frac{d}{d \epsilon} \int_{0}^{1} L\left(q_{1}+h \int_{0}^{u} V(s)+\epsilon \delta V(s) d s, V(u)+\epsilon \delta V(u)\right) d u \\
& =\int_{0}^{1}\left(\frac{\partial L}{\partial q}(Q(u), V(u)) h \int_{0}^{u} \delta V(s) d s+\frac{\partial L}{\partial v}(Q(u), V(u)) \delta V(u)\right) d u \\
& =\int_{0}^{1} \int_{s}^{1} h \frac{\partial L}{\partial q}(Q(u), V(u)) \delta V(s) d u d s+\int_{0}^{1} \frac{\partial L}{\partial v}(Q(u), V(u)) \delta V(u) d u \\
& =\int_{0}^{1} \int_{u}^{1} h \frac{\partial L}{\partial q}(Q(s), V(s)) \delta V(u) d s d u+\int_{0}^{1} \frac{\partial L}{\partial v}(Q(u), V(u)) \delta V(u) d u \\
& =\int_{0}^{1}\left(\int_{u}^{1} h \frac{\partial L}{\partial q}(Q(s), V(s)) d s+\frac{\partial L}{\partial v}(Q(u), V(u))\right) \delta V(u) d u
\end{aligned}
$$

from which

$\nabla S_{h}=\frac{\partial L}{\partial v}(Q(u), V(u))+h \int_{u}^{1} \frac{\partial L}{\partial q}(Q(s), V(s)) d s, \quad Q(u)=q_{1}+h \int_{0}^{u} V(s) d s$.

By the same reasoning as was used to find the differentiability of $S_{h}$, the gradient $\nabla S_{h}$ (best thought of as a vector field) is a $C^{r-1}$ map from $C^{k}\left([0,1], \mathbb{R}^{n}\right)$ to $C^{0}\left([0,1], \mathbb{R}^{n}\right)$, also irrespective of the value of $k, 0 \leq k \leq r-1$.

The constraint of (2) (i.e. the second equation) is $C^{\infty}$ because it is bounded linear, and its derivative is

$$
\delta V(u) \mapsto \int_{0}^{1} \delta V(u) d u .
$$

The kernel of this derivative, say $\mathbb{E}_{0}$, is the tangent space to the constraint set, and it splits $C^{k}\left([0,1], \mathbb{R}^{n}\right)$ orthogonally with respect to the metric $\langle\langle\rangle$,$\rangle (the$ complement is the subspace of constant functions) by

$$
\delta V(u)=\left(\delta V(u)-\int_{0}^{1} \delta V(u)\right) \oplus \int_{0}^{1} \delta V(u)
$$

$S_{h}$ has a critical point on the level sets of the constraint if and only if the orthogonal projection $\mathbb{P}_{\mathbb{E}_{0}}$ of $\nabla S_{h}$ to the kernel $\mathbb{E}_{0}$ is zero i.e. for the solutions to the constrained variational problem (2), one solves

$$
\mathbb{P}_{\mathbb{E}_{0}} \nabla S_{h}\left(V_{0} \oplus V_{1}\right)=0
$$


for $V_{0}$ near

$$
V_{0}=0, \quad V_{1}=0, \quad z=0, \quad q_{1}=\bar{q}, \quad h=0 .
$$

To use the implicit function theorem, one requires that the appropriate partial derivative of $\mathbb{P}_{\mathbb{E}_{0}} \nabla S_{h}$ is a linear isomorphism. Remembering to set $h=0$, that derivative is

$$
\begin{aligned}
\left.\frac{d}{d \epsilon}\right|_{\epsilon=0} \mathbb{P}_{\mathbb{E}_{0}} \frac{\partial L}{\partial v}\left(\bar{q}, \epsilon \delta V_{0}(u)\right) & =\mathbb{P}_{\mathbb{E}_{0}} \frac{\partial^{2} L}{\partial v^{2}}(\bar{q}, 0) \delta V_{0}(u) \\
& =\frac{\partial^{2} L}{\partial v^{2}}(\bar{q}, 0) \delta V_{0}(u)-\int_{0}^{1} \frac{\partial^{2} L}{\partial v^{2}}(\bar{q}, 0) \delta V_{0}(u) \\
& =\frac{\partial^{2} L}{\partial v^{2}}(\bar{q}, 0) \delta V_{0}(u)
\end{aligned}
$$

If $L$ is regular, this is a linear isomorphism of $\mathbb{E}_{0}$, with inverse

$$
\delta V_{0}(u) \mapsto\left(\frac{\partial^{2} L}{\partial v^{2}}(\bar{q}, 0)\right)^{-1} \delta V_{0}(u) .
$$

Thus, the implicit function theorem provides neighborhoods $W_{1} \subseteq \mathbb{R}^{n} \times \mathbb{R}^{n} \times$ $\mathbb{R}=\left\{\left(q_{1}, z, h\right)\right\}$ containing $(\bar{q}, 0,0)$ and $W_{2} \subseteq C^{k}\left([0,1], \mathbb{R}^{n}\right)$ of the constant map $u \mapsto 0$, and a $C^{r-1}$ map $\psi: W_{1} \rightarrow W_{2}$ such that for all $\left(q_{1}, z, h\right) \in W_{1}$, $\psi\left(q_{1}, z, h\right) \in C^{k}\left([0,1], \mathbb{R}^{n}\right)$ is the unique critical point in $W_{2}$ of the constrained variational problem (2). By setting $k=0$ and then $k=r-1$, one can arrange that $W_{2}$ is a $C^{r-1}$ neighborhood, $\psi$ has values in $W_{2}$, and hence in the $C^{r-1}$ curves, $\psi$ is $C^{r-1}$ with the $C^{r-1}$ topology, but that $\psi$ provides the unique solution among the $C^{0}$ curves in a $C^{0}$ open neighborhood, say

$$
\{V(u):|V(u)|<\epsilon\},
$$

of the constant curve 0 .

Now reverse the regularization. Pick an $h>0$ such that $(\bar{q}, 0, h) \in W_{1}$, set

$$
\bar{W}_{1}=\left\{\left(q_{1}, q_{2}\right):\left(q_{1},\left(q_{2}-q_{1}\right) / h, h\right) \in W_{1}\right\}
$$

and define

$$
\bar{\psi}_{\left(q_{1}, q_{2}\right)}(t)=q_{1}+\int_{0}^{\frac{t}{h}} \psi\left(q_{1}, \frac{1}{h}\left(q_{2}-q_{1}\right), h\right)(u) d u
$$

Then $(\bar{q}, \bar{q}) \in \bar{W}_{1}, \bar{\psi}_{\left(q_{1}, q_{2}\right)}(t)$ is defined for $\left(q_{1}, q_{2}\right) \in \bar{W}_{1}$ and $t \in[0, h]$, and $\bar{\psi}_{\left(q_{1}, q_{2}\right)}(t)$ is a first order curve in $T \mathcal{Q}$ which has base integral curve a Lagrangian evolution. This evolution is unique among the continuous curves corresponding to $|V(u)|<\epsilon$ i.e. among $C^{1}$ curves $q(t)$ such that $\left|q^{\prime}(t)\right|<\epsilon / h$, so $C^{1}$ curves $q(t)$ in some $C^{1}$ neighborhood of the constant curve $\bar{q}$. 


\section{$3 \quad$ Remarks}

The regularization can be formulated in invariant terms on the manifold $\mathcal{Q}$, using a tubular neighborhood of the antisymmetric normal bundle of the diagonal of $\mathcal{Q} \times \mathcal{Q}$ to accomplish the subtraction $q_{2}-q_{1}$. Replacing $(Q(u), V(u))$ with its $T \mathcal{Q}$ version $V(u)$, the regularized variational problem, at $h=0$, becomes

$$
S_{0}=\int_{0}^{1} L \circ V, \quad \int_{0}^{1} V(u)=z, \quad \tau_{\mathcal{Q}} V=\text { constant } .
$$

It is a pretty result that the variational principle on $\mathcal{Q}$ regularizes to this trivial one on the fibers of $T \mathcal{Q}$. The map $\bar{\psi}$ is defined only for small $z=\left(q_{2}-q_{1}\right) / h$, and since $\bar{\psi}\left(q_{1}, q_{2}\right)$ is a solution which goes from $q_{1}$ to $q_{2}$ in time $h$, the velocity of this solution is also, approximately, $\left(q_{2}-q_{1}\right) / h$. Thus regularizing only at $z=0$ provides evolutions which correspond only to velocities near zero. This is unacceptable for the objective of solving the initial value problem by first solving the local boundary value problem, because it assigns evolutions only to those initial data corresponding to velocities near zero, while it is known of course from ODE theory that there is a unique integral curve of the Lagrangian vector field corresponding to any velocity. However, minor extensions of the above show that the variational principle actually regularizes at all $z$. The local solutions so obtained along the entire solution (i.e. $z$ maps to the constant curve $V(u)=z$ ) of the regularized variational principle at $h=0$, may be glued together using a technique the can be found for example in [5], page 97 . This provides solutions starting at any velocity. For the same reason, this also an important step in the discrete Lagrangian context $[3$, where the discrete initial value problem is addressed by first solving the local boundary value problem.

\section{References}

[1] R. Abraham and J. E. Marsden. Foundations of Mechanics. AddisionWesley, second edition, 1978.

[2] R. Abraham, J. E. Marsden, and T. S. Ratiu. Manifolds, tensor analysis, and applications. Springer-Verlag, second edition, 1988.

[3] C. Cuell and G. W. Patrick. Discretizations of Lagrangian mechanics. In preparation.

[4] M. Golubitsky and J. E. Marsden. The Morse lemma in infinite dimensions via singularity theory. SIAM J. Math. Anal., 14:1037-1044, 1983.

[5] S. Lang. Differential manifolds. Addison-Wesley, 1972.

[6] G. W. Patrick. Two axially symmetric coupled rigid bodies: relative equilibria, stability, bifurcations, and a momentum preserving symplectic integrator. PhD thesis, University of California at Berkeley, 1991. 
[7] A. J. Tromba. Almost-Riemannian structures on Banach manifolds: the Morse lemma and the Darboux theorem. Canad. J. Math., 28:640-652, 1976.

[8] A. J. Tromba. A sufficient condition for a critical point of a functional to be a minimum and its application to Plateau's problem. Math. Ann., 263:303-312, 1983. 